\title{
Organización Corona: Un caso de innovación exitosa, la baldosa lbérica
}

\section{ESUMEN}

El objetivo de este trabajo es dar a conocer las principales características de un ejercicio de innovación empresarial derivado del diseño y desarrollo de un producto en la cadena productiva de la cerámica en Colombia.

El ejercicio permite conocer de primera mano el proceso de innovación que llevó a cabo la organización, la forma en que se detectó la necesidad y el ejercicio de orientación hacia un segmento de mercado no atendido.

\section{palabras clave}

Innovación, concepto de negocios, nichos de mercado y proceso de manufactura.
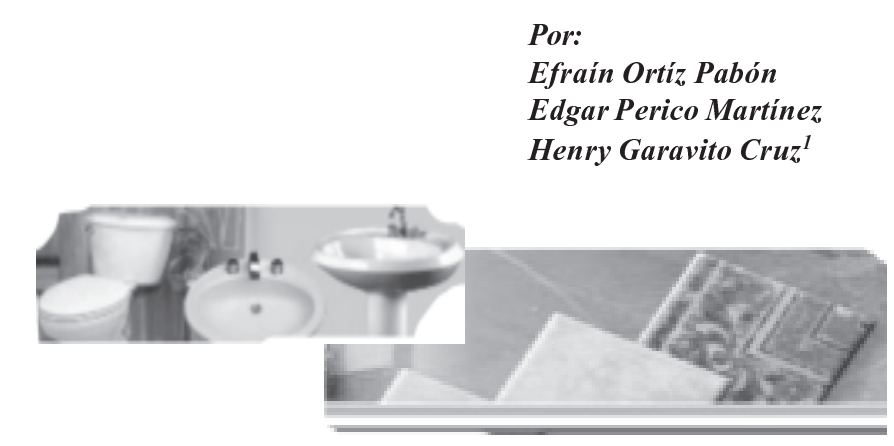

\section{Introducción}

"...Si dejamos de pensar en los pobres como víctimas o como carga, y empezamos a reconocerlos como empresarios creativos $y$ con capacidad de recuperación, y como consumidores con sentido de valor, se abrirá un mundo de nuevas oportunidades...".

\section{C.K. Prahalad}

El objetivo de este trabajo es dar a conocer las principales características de un ejercicio de innovación empresarial derivado del diseño y desarrollo de un producto en la cadena productiva de la cerámica en Colombia.

1 Efraín Ortiz Pabón. Master en Administración de Empresas de la Universidad Politécnica de Madrid, Especialista en Administración de Empresas de la Universidad Politécnica de Madrid, Especialista en Gerencia de Tecnología de la EAN, Ingeniero de Sistemas U. Autónoma. Profesor de la Escuela de Administración de Negocios en Gestión Tecnológica e Iniciativa y Desarrollo Empresarial; Edgar Perico Martínez. Estudiante de Administración de Empresas de la EAN, Jefe de Desarrollo Humano Local UEN Revestimiento de Colcerámica S.A.; Henry Garavito Cruz. Estudiante de Administración de Empresas de la EAN, Director de Mantenimiento Productivo Total, UEN Revestimiento de Colcerámica S.A. 
El ejercicio permite conocer de primera mano el proceso de innovación que llevó a cabo la organización, la forma en que se detectó la necesidad y el ejercicio de orientación hacia un segmento de mercado no atendido.

Aunque la organización Corona por tradición se ha caracterizado por su liderazgo en el mercado de la cerámica y su naturaleza innovadora, pocas veces se le han documentado, por lo menos desde el escenario académico, resultados producto de este tipo de actividades.

La documentación de este caso se hace posible gracias a la intervención directa de dos estudiantes de la Escuela de Administración de Negocios -EAN- en dicho proceso, quienes abordan el ejercicio guiados por el aporte metodológico de un tutor experto en el tema de la innovación.

Los resultados condensados en este documento, parten de una caracterización general de la organización para luego focalizarse en la empresa generadora de la innovación, Colcerámica S.A., y en el producto específico.

\section{Trayectoria de la organización Corona}

La Organización Corona es un grupo empresarial Colombiano, con más de 100 años de tradición en el mercado. Se considera como parte fundamental del patrimonio empresarial latinoamericano y ejemplo de crecimiento y permanente desarrollo para el país.

La historia de esta organización se inicia en 1881 y está ligada a la pujanza y visión de la familia antioqueña Echavarría Olózaga, quienes se concentraron en la creación, operación y expansión de esta organización empresarial dedicada principalmente a la manufactura y comercialización de productos cerámicos.

Como líder empresarial, la Organización Corona ha heredado y fundamentado su éxito en el emprendimiento propio de sus fundadores, característica que le permite erigirse con liderazgo y ejercer como pionero en la generación de innovaciones tecnológicas, y la adopción de principios modernos de gestión empresarial basados en políticas y valores impregnados con un sentido profundo de responsabilidad social.

Esta organización cuenta actualmente con aproximadamente 8.000 empleados y su historia se puede resumir en 5 etapas:

\section{A bStract}

The purpose of this paper is to show the main properties of the innovation process in Baldosa lbérica. This is a complete manufacturing process that includes designing, development and arrangement of the production clusters in the ceramic market in Colombia.

This summary shows a successful process of innovation in a major organization and how it has taken into account of the priorities of an unknown market potential.

\section{MY WORDS}

Innovations, business concept, marketing, manufacturing process. 
Gráfico 1

Estructura actual de negocios de la Organización Corona

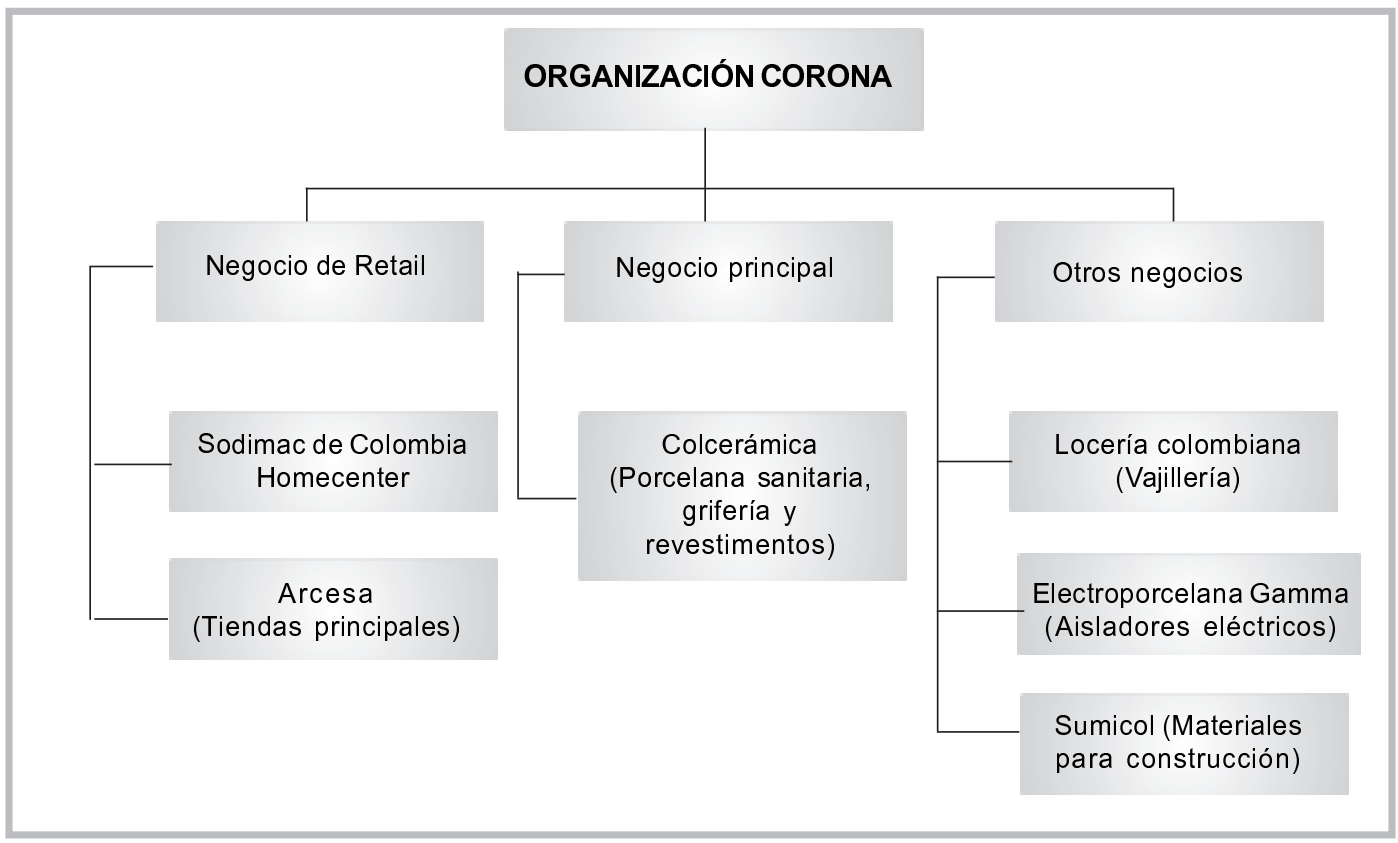

Fuente: Colcerámica

Etapa 1. Nacimiento. (1881-1935) En 1881 se pone en funcionamiento la empresa Cerámica Antioqueña en Medellín como una pequeña planta productora de loza y vidrio inspirada en una actividad artesanal. Para 1906 y debido al comportamiento del mercado se separa el negocio de loza del de vidrio; nacen en Caldas, Locería Colombiana y la Vidriera Caldas, que más tarde se denomina Peldar.

Etapa 2. Enfoque. (1935-1948) En 1935 Gabriel Echavarría Misas adquiere Locería Colombiana la cual se atravesaba por dificultades económicas; se dedica solo a fabricar vajillas y la convierte en la primera empresa de lo que hoy se conoce como la Organización Corona.
Con la compra de la fábrica de lozas por parte de la familia Echavarría se promueve el inicio de la industria cerámica en el país. Desde 1940 y hasta 1947 se marcan hitos importantes en la historia de la compañía debido a la introducción de cambios importantes en los sistemas de producción, políticas de relaciones humanas, actualización tecnológica y sistemas de ventas.

Etapa 3. Expansión. (1948-1990) A partir del año 1948 los accionistas deciden realizar grandes inversiones, se inicia una rápida expansión y se fundan nuevas empresas, la mayor parte de las que hoy conforman la Organización Corona. 
Cuadro 1

Nacimiento y consolidación de las empresas del grupo Corona

\begin{tabular}{|c|c|}
\hline Año & Empresa \\
\hline 1948 & $\begin{array}{l}\text { Creación de Superfrax Ltda., hoy Planta La Estrella de ColCerámica S.A. en Medellín, } \\
\text { para fabricación de mosaico cerámico para revestimientos. }\end{array}$ \\
\hline 1952 & $\begin{array}{l}\text { Se crea en Madrid Cundinamarca, la Compañía Colombiana de Cerámica S.A. } \\
\text { COLCERÁMICA, para la producción de azulejo cerámico. }\end{array}$ \\
\hline 1956 & $\begin{array}{l}\text { 1956, nace Porcelana Sanitaria S.A.- PORSANITARIA, contigua a COLCERÁMICA, } \\
\text { primera planta en el país para la producción de artefactos sanitarios, la que } \\
\text { posteriormente se fusiona con ésta. Se contruye la Planta No. } 1 \text { de baldosa cerámica } \\
\text { para revestimiento de pared de Cerámica Sabaneta S.A. en Antioquia (Hoy Planta } \\
\text { La Estrella). Se inicia la Cadena de Almacenes y Salas para venta al detal de } \\
\text { productos cerámicos. }\end{array}$ \\
\hline 1958 & $\begin{array}{l}\text { Se funda en Barranquilla Lámina del Caribe S.A., con la producción de tableros de } \\
\text { fibra de madera. }\end{array}$ \\
\hline 1960 & $\begin{array}{l}\text { Se crea Electroporcelana Gamma S.A., en Medellín, para producir aisladores } \\
\text { eléctricos y refractarios cerámicos. Inicia actividades Minerales de Colombia S.A., } \\
\text { en La Estrella Antioquia, hoy Suministros de Colombia S.A., para la explotación de } \\
\text { minas de minerales no metálicos y para la investigación y desarrollo de procesos } \\
\text { y productos cerámicos. }\end{array}$ \\
\hline 1962 & $\begin{array}{l}\text { Se constituye en Girardota, Antioquia, Manufacturas de Cerámica S.A. - MANCESA, } \\
\text { hoy la Planta Girardota de ColCerámica S.A., en asocio con American Standard, } \\
\text { para la producción de artefactos sanitarios. }\end{array}$ \\
\hline 1963 & $\begin{array}{l}\text { Se constituye Grifos y Válvulas S.A. - GRIVAL, en Funza, Cundinamarca, hoy Planta } \\
\text { de Grifería de ColCerámica S.A., con la producción de grifería metálica y plástica } \\
\text { para artefactos sanitarios. Se instala en Sabaneta, Antioquia, una planta para la } \\
\text { fabricación de maquinaria cerámica en asocio con Crossley Machines, USA, que } \\
\text { luego se fusiona con Minerales de Colombia para convertirse en Suministros de } \\
\text { Colombia S.A. - SUMICOL S.A., en Sabaneta, Antioquia. }\end{array}$ \\
\hline 1977 & $\begin{array}{l}\text { MANCESA, hoy Planta Girardota de ColCerámica S.A., inicia la producción de } \\
\text { azulejos. }\end{array}$ \\
\hline 1981 & $\begin{array}{l}\text { Se construye una moderna planta de revestimientos para pisos y paredes en Sopó, } \\
\text { Cundinamarca, CERAMITA S.A., hoy la Planta Sopó de ColCerámica S.A. }\end{array}$ \\
\hline 1982 & $\begin{array}{l}\text { Se moderniza la producción de revestimientos con tecnología de una quema rápida } \\
\text { en hornos de rodillos. }\end{array}$ \\
\hline 1994 & $\begin{array}{l}\text { Se conforma "Sodimac Colombia S.A.", en alianza con Sodimac de Chile para } \\
\text { desarrollar la cadena de almacenes Home Center. }\end{array}$ \\
\hline
\end{tabular}

Fuente: www.cecodes.org.co/asociados/colceramica.htm 
Etapa 4. Adaptación a los mercados internacionales. (1990-2000) La Organización Corona se prepara para la apertura económica. Se inician procesos de reestructuración administrativa, se reenfocan los procesos y se implementan agresivos programas de desarrollo humano. La compañía comienza a girar alrededor de conceptos como la administración humanista, la excelencia de la calidad, la importancia máxima del cliente, la participación del personal y el compromiso social. En 1994 se conforma Sodimac Colombia S.A., en alianza con Sodimac de Chile para desarrollar la cadena de almacenes Home Center. En el año 2000 los negocios de porcelana sanitaria, revestimientos y grifería se consolidan como una sola empresa, conformándose el negocio núcleo de la organización con el nombre de Colcerámica S.A.

Etapa 5. Internacionalización. (2000Actual) Se conforma una compañía de comercialización de revestimientos cerámicos enfocada al mercado de los Estados Unidos y se fortalece el mercado en los países andinos y en el Caribe. Se adquiere una compañía de fabricación de porcelana sanitaria y grifería en Estados Unidos. Se fortalecen las empresas Orchid Ceramics y Mancesa Inc. para comercializar productos en Norte América y las alianzas con empresas como Sodimac.

\section{Colcerámica S.A. Como negocio núcleo de la Organización Corona}

Aunque todas las empresas de la Organización Corona se han caracterizado por su empuje, sus procesos estratégicos e innovación, es preciso focalizarnos para este ejercicio en la Compañía Colombiana de Cerámica: Colcerámica S.A. la cual se constituyó como una sola empresa entre los años 2000 y 2001 al fusionarse cinco compañías de la Organización Corona: Ceramita (fundada en 1982), Grival (fundada en 1963), Mancesa (fundada en 1962), Colcerámica (fundada en 1952) y Cerámica Sabaneta (fundada en 1948).

Consolidada la fusión del conjunto de empresas en torno a ColCerámica S.A. el portafolio de productos ascendió a más de catorce mil, los cuales se ofrecen a través de una amplia red de distribuidores en todo el país, Latinoamérica y Estados Unidos. Esta situación hace que la compañía se estructure en torno a tres unidades estratégicas de negocio:

- Porcelana Sanitaria. Enfocada en la fabricación de elementos cerámicos para baño como sanitarios, lavamanos y demás accesorios de porcelana. Cuenta con 1.720 empleados.

- Revestimientos. Enfocada en el mercado de productos cerámicos para recubrimiento de pisos y paredes como baldosas y enchapes cerámicos. Cuenta con 1.550 empleados.

- Grifería. Enfocada en la fabricación y comercialización de grifos, válvulas y accesorios en plástico o fundición que se usan como complemento de la porcelana sanitaria. Cuenta con 580 empleados. 
En la actualidad la empresa esta conformada por siete Centros de Gestión: dos localidades administrativas ubicadas, una en Bogotá y otra en Medellín, y cinco plantas de producción: una planta en Madrid (Cundinamarca) y otra en Girardota (Antioquia) dedicadas a la producción de revestimientos y porcelana sanitaria; una planta en Sopó, que fabrica únicamente revestimientos; una planta en La Estrella (Antioquía) que fabrica accesorios y complementos para los revestimientos y por último una planta en Funza (Cundinamarca) enfocada en la fabricación de grifos y válvulas.

COLCERAMICA S.A. es líder en la fabricación y comercialización de productos cerámicos y de grifería para el hogar. Sus productos son vendidos en Colombia, Ecuador, Venezuela, Centro América y Estados Unidos y ha logrado el posicionamiento de sus marcas CORONA, MANCESA, GRIVAL y, últimamente, ORCHID y MANSFIELD en los Estados Unidos, e IBERICA en Colombia.

\section{Evolución tecnológica de Colcerámica S.A.}

La evolución tecnológica de ColCerámica a lo largo de su historia se fundamenta en cinco frentes: tecnología de maquinaria y equipo, tecnología informática, know how cerámico, tecnología administrativa, tecnología de gestión del conocimiento y sistemas de inteligencia de mercados. Se pueden diferenciar dentro de su evolución algunos períodos con características particulares.

\section{Periodo I: Procesos manuales}

Este período se extiende desde los inicios de la organización hasta la década de los años ochenta. A pesar del rápido crecimiento de ColCerámica, con la expansión acelerada de los negocios y la creación de nuevas empresas, sus modelos correspondían a empresas de la época, caracterizadas porque la mayoría de sus procesos eran de carácter manual, la automatización en maquinaria era mínima tornándose en lentos lo cual se traducía en bajas en la productividad. Era la época de los moldeos y desmoldeos a mano, de los hornos túnel, de las prensas de fricción o de golpe accionadas manualmente, de las decoraciones con calcomanías o hechas a mano, de la selección, empaque y estibado manual, donde el conocimiento estaba en cabeza de pocos y no se requería de sistemas sofisticados para administrar la información.

\section{Periodo II: Automatización}

A partir de la década de los años ochenta se inicia un rápido proceso de automatización, se incrementa de manera ostensible la productividad lo cual exige nuevas formas de administrar. La nueva tecnología de maquinaria que se incorpora, permite fabricar nuevos y diferentes productos, de tal manera que el escaso portafolio que se tenía para la época, es reemplazado por uno muy amplio, que permite nuevas opciones a los clientes y consumidores. En este periodo se da inicio a la implementación de sistemas de información rápidos y confiables, que suministren información en tiempo real tanto de las máquinas como del comportamiento de los procesos. Paralelamente se incorporan ejercicios de desarrollo humano que brindan recursos para afrontar los efectos derivados de la apertura económica que se daría en al país algunos años después.

\section{Periodo III: Expansión tecnológica}

Para la década de los 90, ColCerámica inicia una etapa de transición que le permite incorporar los cambios estructurales y tecnológicos necesarios 
para convertirse en empresa multinacional. Se rediseñan las estructuras, se desarrollan nuevos productos para el mercado externo intensivos en investigación y desarrollo (I+D). Se adquiere nueva tecnología en forma de maquinaria y equipo la cual permite que se desarrollen los nuevos productos.

Se da inicio a la intención de abrir nuevos mercados, se instalan sistemas de información sofisticados que permiten administrar la totalidad del negocio con una cadena de abastecimiento sólida y estructurada la cual se ve reforzada con capital humano de nueva generación y gestores que dan impulso a la organización.

\section{Periodo IV: Consolidación e internacionalización}

A principios del nuevo siglo ColCerámica termina su proceso de estructuración. Da inicio a un proceso de actualizaciones e inversiones en tecnología que le permiten competir a nivel mundial.

Los procesos de innovación alcanzan su más alto nivel de evolución tecnológica con el desarrollo de nuevos productos; se implementan nuevosmodelos administrativos y de gestión, que buscan incrementar la productividad y competitividad; se invierte significativamente en desarrollos tecnológicos orientados hacia la logística y se da inicio al desarrollo de tecnologías propias que le permitan gestionar el conocimiento.

\section{Un caso exitoso de innovación: la línea ibérica}

Para el año 2002 el negocio del revestimiento incluye en su proceso de gestión un reenfoque estratégico consistente en una idea sencilla pero novedosa para la Organización: "Aprovechar el potencial de los mercados populares y desarrollar una línea de productos dirigida a estratos medios y medios - bajos de la sociedad Colombiana". Esto produce un giro importante si se tiene en cuenta que históricamente la organización se concentró en posicionar la marca Corona en mercados de nivel socioeconómico medio y alto. Este nuevo enfoque estratégico lleva al nacimiento de la línea y la marca IBERICA.

\section{El proceso de innovación de la línea IBERICA en el negocio de revestimiento}

La creación, consolidación y expansión de la línea IBERICA, se puede considerar como un ejemplo de innovación exitosa desde diferentes puntos de vista: i) en concepto de negocios, ii) en valor, iii) en producto, iv) en proceso.

Como concepto de negocios ${ }^{2}$ es distintiva ya que el esfuerzo organizacional está dirigido a la base de la pirámide y la fortaleza de este enfoque innovador radica en que busca crear oportunidades

\footnotetext{
2 "Los revolucionarios de la industria asumen todo un concepto del negocio, más bien que un producto o servicio, como punto de partida para la innovación. Reconocen que la competencia ya no es entre productos o servicios sino entre conceptos mercantiles." (Hamel G. 2000).
} 
para los segmentos menos favorecidos al ofrecerles opciones y estimular su autoestima suministrando un producto de calidad que les resulta rentable (Prahalad, 2005).

Indudablemente es una innovación en valor $r^{3}$, el foco de atención para la innovación no choca de frente con los competidores. Se aplica una lógica estratégica diferente consistente en abrir un espacio nuevo en el mercado y se logra un salto cualitativo en valor tanto para los compradores como para la compañía. La lógica de este tipo de innovaciones radica fundamentalmente en que se reducen costos y se eleva simultáneamente el valor para los compradores ( Cham Kim, et al., 2005).

Es una innovación en producto ${ }^{4}$ porque se vale de la experiencia acumulada a través de muchos años de esfuerzo e investigación, que al traducirse en la generación de conocimiento tácito para la compañía, sirve de base y permite el desarrollo de un producto nuevo con especificaciones de calidad similares a la marca Corona. Este producto se orienta a un nicho de mercado no explotado y obtiene éxito comercial sin alterar el foco de mercado de la marca base, por el contrario, fortalece su prosicio-namiento en los sectores socioeconómicos medio y alto.
Es una innovación en proceso 5 porque está destinada a mejorar las capacidades de rapidez, el perfeccionamiento de los materiales existentes, la agilidad y la calidad de la empresa (El-Hadj, 1990). Se sustenta en una mejora técnica derivada de la capitalización de la experiencia obtenida a los largo de muchos años que sumada a la creatividad e investigación y desarrollo permanentes en el sector cerámico, dan forma sistémica al proceso.

Al visualizar en detalle este ejercicio, se identifica que la compañía centra el proceso innovador en tres momentos principales: iniciación, articulación y promoción; igualmente, el éxito radica en el manejo holístico que se da en cada una de sus fases, y cada una de las actividades que se desarrollan está perfectamente alineada y articulada de comienzo a fin (Ortiz y Nagles, 2005).

A continuación se explica de forma general cómo interviene cada momento de la innovación, y en el apéndice metodológico se puede visualizar en detalle la forma en que se interactúa dentro del proceso innovador.

\footnotetext{
3 "La innovación en valor se crea en la región en la cual los actos de una compañía inciden favorablemente sobre su estructura de costos y sobre la propuesta de valor para los compradores. Las economías se logran al eliminar y reducir las variables sobre las cuales compite la industria. El Valor para los compradores se aumenta al buscar y crear elementos que la industria nunca ha ofrecido. Con el tiempo, los costos se reducen todavía más cuando entran a operar las economías de escala debido al mayor nivel de ventas emanado del valor superior." (Cham Kim, et al., 2005)

4 "La innovaciones de producto o innovaciones externas permiten a las empresas ofertar mejores productos que los existentes en el mercado, ya que ofrecen más funcionalidades, o cumplen funciones de manera más eficaz siendo más ligeros, menos voluminosos, más sencillos, etc. También se puede tratar de productos totalmente nuevos en el propio concepto." (El-Hadj, 1990).

5 "Este tipo de innovaciones se basan a menudo en una mejora técnica de los procesos materiales de producción, ya sea mediante la inversión, por el perfeccionamiento de los materiales existentes, o por una capitalización de la experiencia" (El-Hadj, 1990).
} 


\section{El momento de iniciación de la innovación}

IBERICA nace como una estrategia de mercado (Anexo 1) enfocada a solucionar una discrepancia que se presenta entre los productos de la competencia y los productos de la marca Corona cuando se trata de capturar clientes con baja disponibilidad de capital. La competencia ofrece productos de calidad aceptable, pero con precios muy bajos; Corona ofrece excelente calidad, pero precios altos. Lo anterior obliga permanentemente al área de mercadeo de ColCerámica a desarrollar estrategias como rebajas, descuentos y promociones, que no le generan ningún margen a la compañía y que desgastan la marca Corona, la cual viene ya posicionada como una marca de prestigio en el mercado colombiano.

Al verificar quienes son los consumidores objetivo de los productos de bajo costo, ColCerámica encuentra que corresponden a personas de los estratos 2 y 3 , a quienes les interesa recubrir sus pisos, pero no les alcanza el presupuesto para comprar cerámica a precios altos. Se establece entonces, que hay un mercado potencial al cual se le debe diseñar un producto específico. Se inicia un proceso de validación de la idea, que lleva a la realización de estudios de mercado estructurados, los cuales finalmente corroboran la necesidad que tiene el mercado llegando al nacimiento de la línea IBERICA.

\section{El momento de articulación de la innovación}

El concepto de la línea IBERICA, comienza a desarrollarse desde las diferentes gerencias del negocio lideradas por el área de mercadeo.

El negocio de revestimiento de ColCerámica se enfrenta a la disyuntiva de desarrollar un producto para un mercado desconocido sin afectar el posicionamiento y prestigio que tienen sus propias marcas en otros públicos. Al desarrollar el concepto, se inicia la planeación e implementación de cada uno de los procesos asociados con la operacionalización del nuevo producto. Se ponen en blanco y negro cada una de las iniciativas requeridas para hacer realidad esta nueva estrategia. Se establecen elementos diferenciadores de los productos y los servicios, se hacen los diseños, se establecen las formas de producción y comercialización y se da comienzo a un proceso de cambio cultural en los empleados que inicialmente se verían involucrados en el desarrollo, fabricación, mercadeo y venta de los primeros lotes de producción.

Los productos de la marca IBERICA se comienzan a producir en octubre de 2003 con 33 referencias color: 21 referencias en paredes y 12 para pisos. Se toma la decisión de concentrar la planta de revestimiento ubicada en Madrid, Cundinamarca, para que se dedique exclusivamente al desarrollo de estos productos.

Desde el punto de vista técnico, los productos siempre se han caracterizado por ser productos que cumplen las normas nacionales e internacionales de calidad. Se ensamblan con un nivel medio de decoración y un diseño más competitivo que los productos similares del mercado, pero con menores especificaciones que los productos marca Corona. Tanto para pisos como para paredes, se fabrican versiones brillantes y semibrillantes con protecciones al tráfico que los hacen más duraderos que los de la competencia. Sin embargo, el imperativo más importante de la estrategia IBERICA ha estado en la disminución de los costos: se busca ofrecer un precio consistentemente más bajo sin perder margen. 
Lograr desarrollar la línea IBERICA requirió que desde un principio se tuvieran que definir criterios absolutamente claros en todas las áreas de la compañía, pero principalmente en manufactura, logística y comercialización.

Manufactura. Cumplimiento de las especificaciones señalas en NTC 919 y 4321; organización administrativa regida por los criterios de eficiencia en el uso de los recursos; alta productividad de la mano de obra; calidad de acuerdo con especificaciones técnicas; costo unitario y entregas oportunas; mínimos cambios de referencias, producción en lotes grandes; continuos planes de mejoramiento e innovación; estricto control de inventarios en insumos y repuestos de maquinaria y reaprovechamiento de insumos y productos en proceso.

Logística. Desarrollo de alianzas estratégicas con proveedores $y$ transportadores enfocadas al logro de costos bajos; productos entregados contra disponibilidad; posibilidad de consumir insumos obsoletos o de baja rotación de inventario; almacenamiento en planta y redes de distribución eficientes y económicas y estricto control de inventarios de producto terminado.

Mercadeo y ventas. Mercado enfocado (segmento medio y bajo); la oferta debe ser un diseño atractivo a un precio muy bajo; puntos de venta económicos y populares; un año de garantía (aplicando restricciones); no se invierte en soporte de marca; servicios de acuerdo al mercado objetivo y bajas inversiones en publicidad.

\section{El momento de promoción de la innovación}

El plan de mercadeo y ventas incluyó dos meses con pruebas de campo en el mercado. Se establecieron mecanismos de seguimiento, medición y parámetros de comparación. Durante este periodo de tiempo se pudo vislumbrar el éxito de la línea y se lograron establecer las oportunidades de mejoramiento de la innovación. Se hicieron los ajustes necesarios para proceder a la fabricación de lotes grandes de producción y el lanzamiento de la línea en los mercados objetivo. Posteriormente el negocio ha implementado procesos de mejora continua que no solo han sostenido la línea en el mercado, sino que la han fortalecido y la están proyectando de forma sorprendente.

\section{Los resultados de la innovación}

Ventas. Desde el planteamiento hipotético de la estrategia, las ventas son definidas como elemento fundamental de esa comprobación. Inicialmente se hicieron presupuestos bajos de ventas que rápidamente fueron superados por la realidad. Se inicia con unas ventas inferiores a las 100.000 unidades y en el término de tres meses son triplicadas $y$ al cabo de los seis meses son cinco veces mayores que las ventas iniciales, superando cualquier expectativa. 
Gráfico 2

Evolución en las ventas de la línea IBERICA desde su lanzamiento

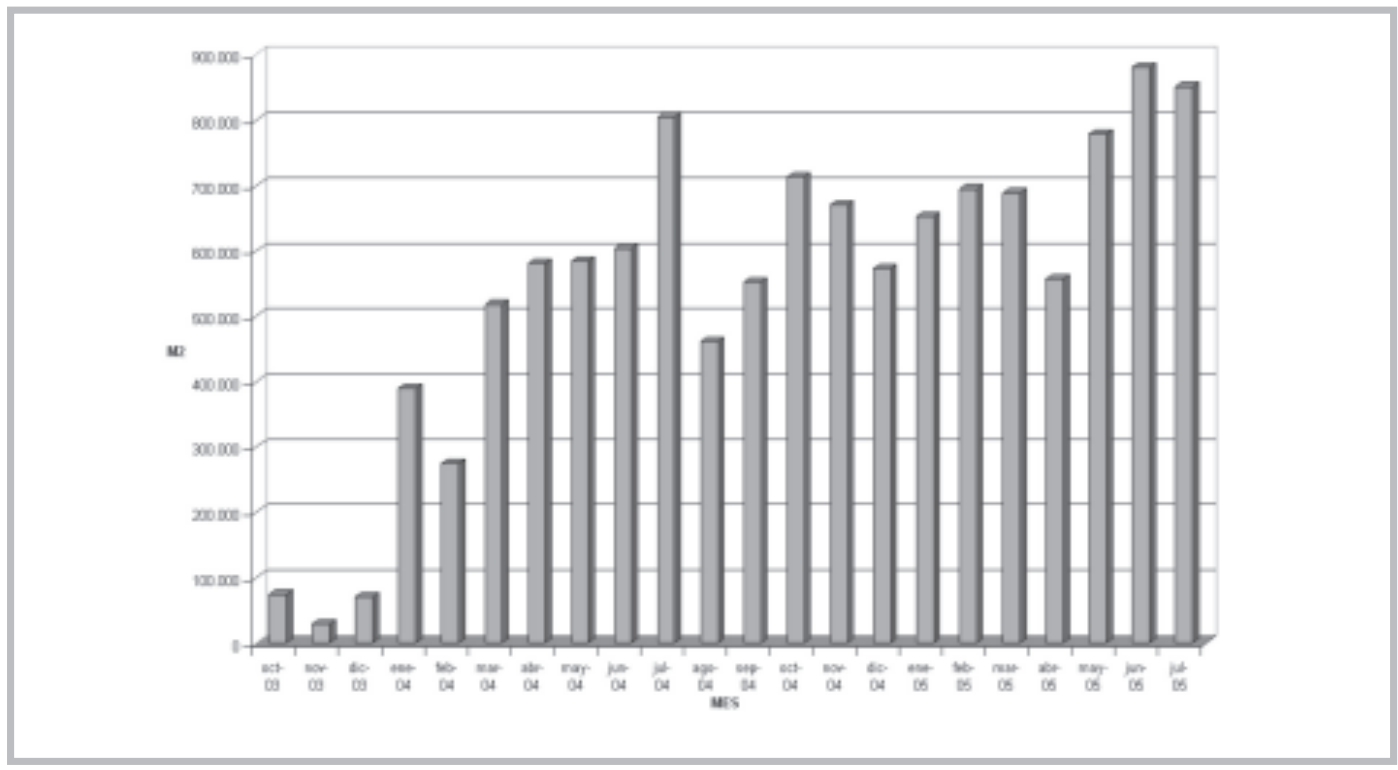

Fuente: Colcerámica S.A.

Con el objetivo de conocer la opinión, percepción, fortalezas, amenazas y satisfacción de clientes, se realizan encuestas de satisfacción que dan como resultado que los productos son acordes con el segmento objetivo, que se han generado mayores volúmenes de ventas en los negocios distribuidores y se reconoce que el precio ha sido su mayor impulsor.

Producción. La producción se ha venido ajustando a la creciente demanda del producto y ha requerido ingentes esfuerzos del área de manufactura para adaptarse rápidamente a las exigencias del nuevo mercado. La dirección de esta área ha debido hacer cambios drásticos tanto en la focalización de sus instalaciones fabriles como en sus procesos administrativos a fin de satisfacer los pedidos de los clientes dentro de los plazos convenidos. En el cuadro 1 se mencionan los principales ajustes en elementos del proceso que han suscitado innovación en el caso IBERICA, y que ha tenido que desarrollar la compañía en el área de producción.

Como se observa en la gráfica de producción, ésta ha tratado de seguir los pedidos de los clientes pero se ha venido quedando corta de tal manera que se ha generado un volumen importante de pedidos pendientes que han obligado al negocio a pensar en nuevas inversiones para satisfacer la demanda. 
Cuadro 1

Procesos, ajuste e innovaciones en la producción de la línea IBERICA

\begin{tabular}{|l|l|}
\hline \multicolumn{1}{|c|}{ Proceso intervenido } & \multicolumn{1}{c|}{ Ajustes e innovaciones } \\
\hline Preparación de la pasta & $\begin{array}{l}\text { - Reformulación de la pasta. } \\
\text { - Rediseño y reubicación de maquinaria. }\end{array}$ \\
\hline $\begin{array}{l}\text { Preparación de esmaltes y preparación de } \\
\text { serigrafías }\end{array}$ & $\begin{array}{l}\text { - Reformulación de esmaltes } \\
\text { - Reformulación de serigrafías } \\
\text { - Rediseño y reubicación de maquinaria }\end{array}$ \\
\hline Prensado, esmaltado, decorado y cocción & $\begin{array}{l}\text { Ajustes en tecnologías administrativas para: } \\
\text { - Incrementar la productividad. } \\
\text { - Rejorar las entregas. } \\
\text { - Disminuir los costos de mantenimiento. } \\
\text { - Reestructuración y reorganización de equi- } \\
\text { pos de trabajo. }\end{array}$ \\
\hline Empaque & $\begin{array}{l}\text { - Cambio de litografías } \\
\text { - Cambios en la modulación. } \\
\text { - Cambios en la logística de transporte. }\end{array}$ \\
\hline
\end{tabular}

Fuente: Colcerámica S.A.

Gráfico 3

Evolución en la producción de la línea IBERICA desde su lanzamiento

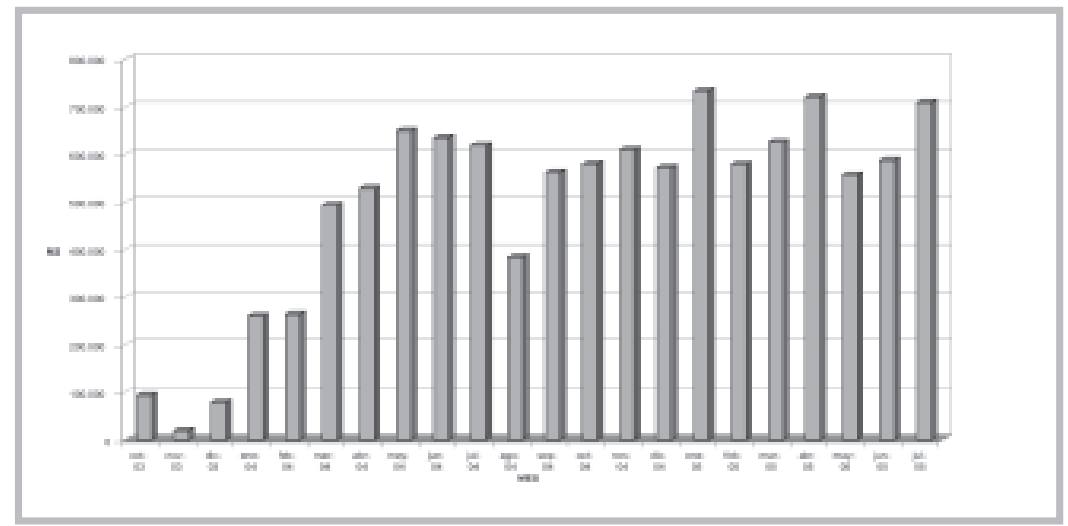

Fuente: Colcerámica S.A.

Proyecciones. A pesar de que la compañía ha hecho un excelente trabajo para responder a la demanda, la línea IBERICA ha entrado en un punto donde la oferta no está cubriendo la demanda del producto, y los estudios del área de mercadeo muestran unas proyecciones de ventas aún mayores para los años siguientes. Esto ha obligado a pensar en la forma como se va a atender esa demanda creciente. Como resultado de esta situación el gobierno corporativo de la Organización Corona, ha aprobado nuevas inversiones que entrarán en operación el año siguiente y que refuerzan la confianza de los accionistas en el éxito del planteamiento estratégico. La Unidad Estratégica de Revestimiento de Colcerámica S.A., está enfocando sus inversiones hacía una línea de producto y hacia unos mercados que hace tres años, nadie se imaginaba que irían a existir. 


\section{Conclusiones}

La importancia de la industria de la cerámica radica esencialmente en el estrecho vínculo que tiene con el sector de la construcción; de allí se derivan los productos empleados en el revestimiento de pisos y paredes y en productos de porcelana sanitaria. El sector cerámico en Colombia está altamente concentrado; más del $80 \%$ de la producción pertenece a cuatro empresas: Colcerámica S.A. (Grupo Corona), Cerámica Italia (10\% del mercado de pisos en el país), Alfagrés S.A. (nacional) y Eurocerámica (nacional).

De acuerdo con la caracterización de las cadenas productivas, ejercicio abordado durante varios años por el DNP (Departamento Nacional de Planeación), se puede constatar que el sector viene siendo sometido a una competencia externa que se suma a un indicador de insuficiencia en la oferta nacional; no obstante la balanza comercial de la cadena de la cerámica, para el periodo 2001-2003, presentó un superavit promedio anual de U\$36 millones.

Efectos como los anteriores, aunados a la política nacional orientada a estimular la construcción, el nacimiento de nuevas líneas de crédito para financiación de vivienda de interés social, además de los subsidios en tal sentido, dejan ver que la orientación estratégica seguida por Colcerámica S.A. al lanzar un nuevo producto, hace reaccionar un mercado no cubierto, el cual se siente atraído frente a la calidad y los bajos precios.
En este contexto se corrobora que la innovación tiene un momento (el auge de la construcción) y explota una oportunidad (la política nacional), pero que su ejercicio de articulación exige experiencia (más de cien años en el mercado), capacidad de reacción organización) y sentido de diferenciación (precio y valor para el comprador).

Pero la oportunidad para el innovador no llega sola, es necesario sumarle otros elementos que lo pueden potenciar aún más, como son: la competitividad y experiencia del sector cerámico colombiano en los mercados internacionales, la posibilidad de expandir su mercado geográfico a nichos similares de países cercanos, la calidad de su producción, la firma de tratados internacionales y la creatividad permanente originada en su cultura y tradición emprendedora. Este último es quizá uno de los principales aspectos de este modelo de innovación, la oportunidad en la reacción del emprendedor, la forma como explota su capacidad de reacción y la forma en que se asume el riesgo en aras de la novedad.

Finalmente, un aspecto importante son las nuevas capacidades, el nuevo conocimiento logrado mediante la línea IBERICA en cada uno de los aspectos enunciados, los cuales necesariamente beneficiarán por defecto no solamente a la línea Corona, sino también a otras en el futuro dentro de la organización. 


\section{Bibliografía}

AFUAH, Allan (1997). La dinámica de la innovación organizacional. Oxford, México.

BETZ, Frederick (1998). Managing technological innovation. Jhon Wiley \& Sons, Inc. NY.

CHAN KIM, W.; MAUBORGNE, Renée (2005). La estrategia del océano azul. Grupo Editorial Norma - Harvard Business School Press, Bogotá.

CHRISTENSEN, Clayton; RAYNOR, Michael (2003). La solución de los innovadores. McGrawHill, Madrid.

DRUCKER, Peter (2002). Escritos fundamentales. Tomo 1, Sudamericana, Buenos Aires.

EL-HADJ, Smail Ait (1990). Gestión de la tecnología. Addisson Wesley-Gestión 3000, Barcelona.

ESCORSA, Pere; VALLS, Pasola (2001). Tecnología e innovación en la empresa. Alfaomega, Bogotá.

HAMEL, Gary (2000). Liderando la Revolución. Grupo Editorial Norma - Harvard Business School Press, Bogotá.

KUCSMARSKI, Thomas D. (1997). Innovación. McGrawHill, Bogotá.

LEONARD, Dorothy (1998). Wellsprings of Knowledge. HBSP, Boston.

MANDADO, Enrique; FERNANDEZ, Francisco J.; DOIRO Manuel (2003) La innovación tecnológica en las organizaciones. Thomson, Madrid.

MILLER, William; MORRIS, Langdon (1999). 4TH Generation R\&D. Jhon Wiley \& Sons, NY.

ORTIZ, Efraín; NAGLES, Nofal (2005). Gestión de la Tecnología, la creatividad y la innovación. (Texto en redacción) Escuela de Administración de Negocios - EAN.

PRAHALAD, C.K. (2005). La oportunidad de negocios en la base de la pirámide. Grupo Editorial Norma - Wharton School Publishing, Bogotá.

SANTESMASES M., Miguel (1999). Marketing conceptos y estrategias. Pirámide -ESIC, Madrid.

ULRICH, Karl T.; EPPINGER, Steven D. (2004). Diseño y desarrollo de productos. McGrawHill, México.

UTTERBACK, James M. (1994) Mastering the Dynamics of Innovation. HBSP, Boston.

VARGAS, Marisela; MALAVER, Florentino; ZERDA, Álvaro (2003). La innovación tecnológica en la industria Colombiana. Ceja Centro Editorial Javeriano, Bogotá.

CECODES. www.cecodes.org.co/asociados/colceramica.htm 


\section{ANEXO 1}

\section{Análisis de la industria y segmentación del mercado}

En este sentido el ejercicio desarrollado por la compañía consistió principalmente en analizar quién es el consumidor y cuál es la segmentación del mercado nacional; esto con el fin de determinar hacia donde enfocar los esfuerzos y con qué tipo de producto incursionar.
Con el conocimiento de la industria asociado al negocio se establece la segmentación del mercado para identificar claramente el foco de oportunidad y las características básicas de ese foco y determinar las hipótesis de trabajo.

Cuadro 2

Segmentación del mercado

\begin{tabular}{|c|c|c|c|c|}
\hline \multirow[b]{2}{*}{$\begin{array}{l}\text { Nivel socio } \\
\text { económico }\end{array}$} & \multicolumn{2}{|c|}{$\begin{array}{c}\text { Cantidad de } \\
\text { personas }\end{array}$} & \multicolumn{2}{|c|}{$\begin{array}{l}\text { Distribuciòn } \\
\text { de viviendas } \\
\text { urbanas** }\end{array}$} \\
\hline & Miles & $\%$ & Cantidad & $\%$ \\
\hline ESTRATO 6 & 1'338 & 3 & 93.922 & 2,8 \\
\hline ESTRATO 5 & 2'229 & 5 & 167.952 & 5,0 \\
\hline ESTRATO 4 & 4’458 & 10 & 304.381 & 9,1 \\
\hline ESTRATO 3 & $18^{\prime} 280$ & 41 & 1'054.962 & 31,5 \\
\hline ESTRATO 2 & $13 ' 821$ & 31 & 1'160.416 & 34,6 \\
\hline ESTRATO 1 & $4^{\prime} 458$ & 10 & 568.004 & 17 \\
\hline TOTALES & $44^{\prime} 584$ & 100 & 3'349.707 & 100 \\
\hline
\end{tabular}

Fuente: Colcerámica S.A.

Se ve un altísimo potencial en los estratos tres bajo y estrato dos que corresponden a más del $50 \%$ de las viviendas urbanas, personas que buscan complementar su vivienda y cuyo principal determinante al momento de comprar es el precio.

\section{Planteamiento estratégico}

Con la idea clara, se establece el foco de mercado, la propuesta de valor, la disciplina del mercado y su alcance. El equipo responsable de sacar adelante la estrategia IBERICA, se concentra en validar las propuestas e iniciativas que comienzan a surgir, apoyándose en estudios e investigaciones de mercado que los llevan a plantear una primera hipótesis de la forma como se desarrollará la nueva línea de producción. 


\section{Definiciones de la estrategia para su implementación}

Enfoque. Consumidores cuyo interés básico es el precio sin importar la marca.

Consumidor que remodela o complementa su vivienda basado en sus necesidades funcionales, higiénicas y de diseño.

Mercado objetivo. Propietarios de vivienda del segmento medio-bajo y bajo que buscan soluciones para complementar su vivienda al precio más bajo y proyectos de vivienda del segmento medio - bajo y bajo, cuyo principal criterio de decisión de compra es el precio.

\section{Elementos clave:}

- La solución más económica del mercado.
- Diseño adecuado al segmento objetivo.

- Comercialización eficiente.

- Manufactura local e instalaciones focalizadas.

- Mediana concentración de clientes con volumen bajo.

- Eficiencia operativa centrada en el control de los costos.

- Estrategias y marcas diferenciadas de los productos Corona. 
Cuadro 3

Planteamiento estratégico para la línea IBERICA

\begin{tabular}{|l|}
\multicolumn{1}{c|}{$\begin{array}{c}\text { Hipótesis : Mercado masivo } \\
\text { Definiciones de valor para el cliente }\end{array}$} \\
\hline Foco de mercado: \\
- Propietario de vivienda del segmento medio-bajo y bajo (estrato 2, parte del 3), que buscan soluciones \\
para complementar su vivienda.
\end{tabular}

Fuente: Colcerámica S.A. 


\section{Apéndice metodológico 6}

\section{El proceso innovador}

El proceso innovador se puede estructurar en tres (3) momentos: i) iniciación, ii) articulación, iii) promoción.

Gráfico 1

El proceso de innovación

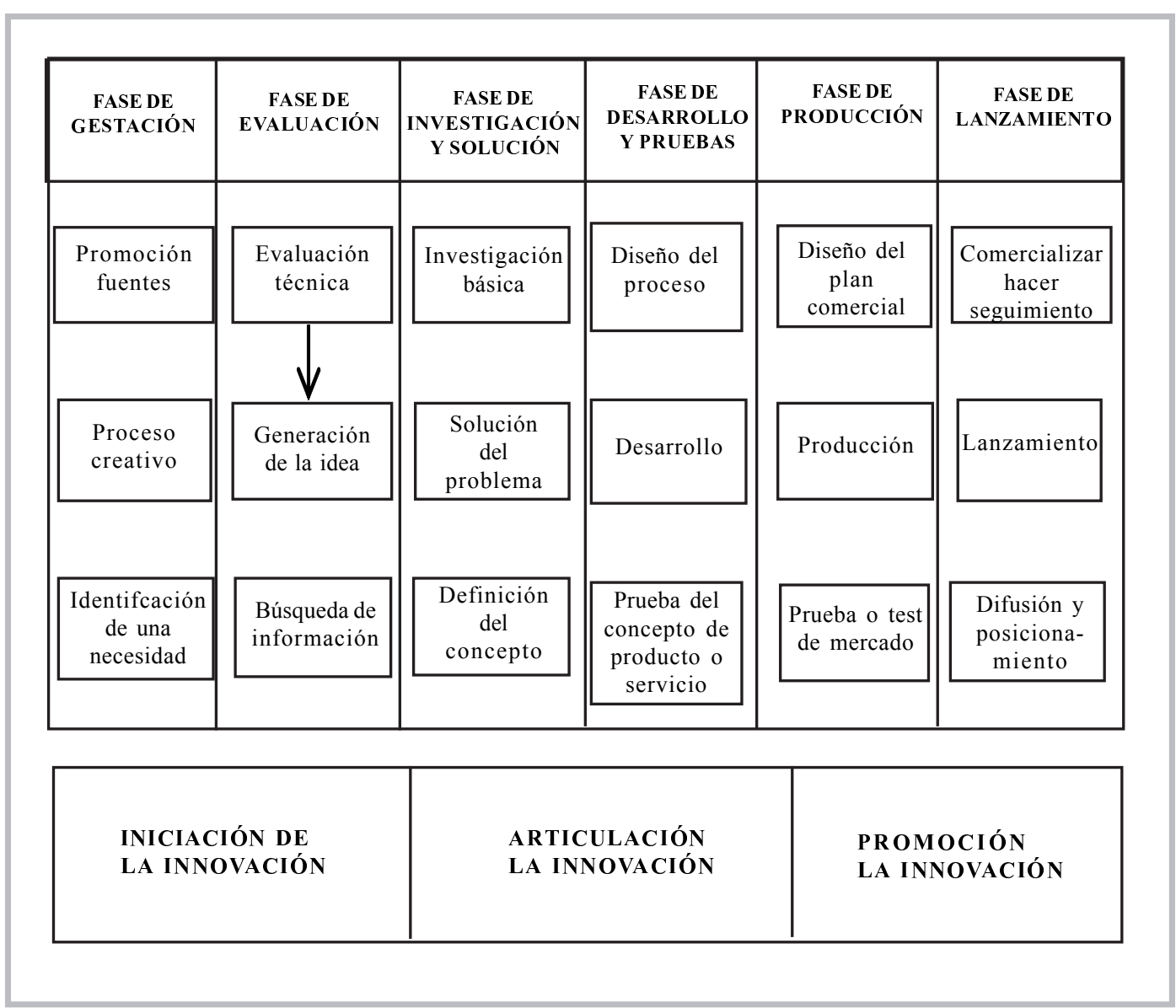

Fuente: Ortíz y Nagles (2005)

\footnotetext{
6 Este apéndice metodológico es extractado del Libro Gestión de la Tecnología y La innovación en fase de corrección luego de haber pasado la revisión técnica, autoría de: Efrain Ortiz Pabón y nogal Nagles García, el cual será publicado próximamente por la EAN.
} 
El momento de iniciación hace referencia al conjunto de actividades propias del proceso creativo que dan lugar a la generación de una idea potencialmente innovadora. Este nivel se encuentra constituido por dos fases: gestación y evaluación.

Durante la fase de gestación es necesaria la promoción del sentido innovador que no es otra cosa que dar lugar a que el espíritu emprendedor (entrepreneurship) disponga de la identificación de fuentes de ideas y necesidades.

Para Drucker (2002) hay potencialidad en siete fuentes de ideas innovadoras: i) los éxitos inesperados y los fracasos inexplicados de la misma organización, pero también los éxitos inesperados y los fracasos inexplicados de la competencia; ii) las incongruencias, especialmente incongruencias en el proceso (ya sea de producción o distribución) o en el comportamiento del consumidor; iii) las necesidades del proceso; iv) los cambios demográficos, v) los cambios en el significado y la percepción; vi) los cambios en la estructura del mercado y de la industria; vii) los conocimientos nuevos.

Para que la fase de gestación cobre vigencia, es necesario acompañarla de una fase de evaluación la cual se constituye en el eslabón que genera y fortalece las ideas dentro de la cadena. La mejor forma de validar esta fase, es dotando la idea de información valiosa que la nutra y la dibuje como viable, para lo cual será necesario contemplar una o más soluciones, frente a una o más necesidades, de uno o más clientes. La idea en esta parte del proceso debe terminar suscitado gusto, interés y positivismo para el emprendedor o quienes la contemplen.
Del momento de articulación de la innovación hacen parte las fases de investigación y solución, desarrollo y pruebas. Su objetivo es proveer de los elementos de investigación básica, definición del concepto de producto o servicio, diseño de proceso y pruebas necesarios para hacer que la innovación cobre vigencia desde el punto de vista formal.

La investigación básica dentro del proceso innovador tiene como función adquirir los conocimientos científicos sobre los fundamentos de los fenómenos y hechos observables tales como propiedades, estructuras y relaciones de la idea en cuestión (Escorsa, et al., 2001). De otra parte el concepto de producto es una descripción aproximada de la tecnología, principios de funcionamiento, y forma del producto o servicio. Es una descripción concisa sobre cómo va a satisfacer el producto o servicio las necesidades del cliente. Por lo general, un concepto se expresa como un bosquejo o un modelo tridimensional tosco, y con frecuencia es acompañado por una breve descripción textual (Ulrich, et al., 2004).

Desde la perspectiva de la fase de desarrollo y específicamente en cuanto al diseño de proceso, es necesario precisar que una innovación será viable en la medida que el conjunto de actividades que dan lugar a su desarrollo (producto o servicio) estén interrelacionadas y orientadas a obtener el resultado específico agregando un valor considerable que pueda ser percibido por el cliente y por quien la genera. En términos prácticos, el diseño coherente de un proceso ${ }^{7}$ o su rediseño permiten reducir o eliminar costos a partir de la reducción en el número de pasos en el proceso de fabricación.

\footnotetext{
7 En áreas como el diseño industrial se consideran productos con proceso intensivo, aquellos en los cuales el proceso de producción impone restricciones estrictas en las propiedades del producto, por lo que el diseño de éste no se puede separar, incluso en la parte de concepto, del diseño del proceso de producción. Ejemplo: semiconductores, alimentos, químicos y papel (Ulrich, et al., 2004).
} 
En cuanto a las pruebas del concepto de producto o servicio Ulrich, et al. (2004) establece que se deben llevar a cabo en siente pasos: i) definir el propósito de la prueba, ii) seleccionar la población a encuestar, iii) seleccionar el formato de la encuesta, iv) comunicar el concepto del producto o servicio, v) medir la respuesta del cliente, vi) interpretar los resultados, vii) incorporar los resultados al producto y al proceso. Esta parte del ejercicio es importante como lo define Escorsa, et al. (2001) porque permite captar información valiosa antes de lanzar el producto al mercado, información que sirve para reformular el concepto si hay lugar a ello o para definir los segmentos que cuenten con los mejores clientes potenciales y de esta forma determinar hacia dónde debe dirigirse el ejercicio posterior del marketing.

El momento de promoción de la innovación abarca las fases de producción y lanzamiento más la difusión y el posicionamiento; están constituidos por el diseño del plan comercial y las pruebas de mercado en primera instancia, que posteriormente dan lugar a la difusión del producto, su comercialización y seguimiento.

Este momento se puede considerar como la primera prueba de fuego de una innovación porque aquí culmina el proceso a través del cual la idea inicial es materializada. Este es el momento propicio para diseñar el plan comercial. Este constituye la acción que permite establecer como serán las relaciones de intercambio con el cliente. A este plan se llega como resultado de la integración con la planificación estratégica que se tenga definida y busca que la oportunidad de negocio sea aprovechada al máximo y pueda hacer frente a los retos que se deriven del mercado.

Las pruebas o test de mercado las plantea Santesmases (1999) como una comercialización real del producto nuevo a escala reducida. Se efectúa en un mercado limitado, de tamaño pequeño, pero representativo del conjunto del mercado al cual va a dirigirse el producto cuando se lleve a cabo su lanzamiento y comercialización definitiva. El test de mercado es una herramienta valiosa porque permite obtener una estimación real de cómo será aceptado el producto por el mercado, y posibilita, por tanto, efectuar modificaciones en la estrategia de marketing o decidir el no lanzamiento definitivo del producto si se observan pobres resultados.

En cuanto a la fase de lanzamiento y difusión es preciso acotar que sólo se hace factible si en todas las etapas anteriores los resultados fueron satisfactorios. Permite comprobar la efectividad de la innovación mediante la aceptación del producto en el mercado. Se pueden establecer dos parámetros iniciales de medición. El primero hace referencia a la aceptación que en el corto plazo tenga el producto, y el segundo al nivel de repetición en la compra que se logre. Es en esta fase donde el producto comprueba si resiste los ataques de los competidores y se mide qué tan preparado está el innovador para reaccionar. No solo se pone a prueba el producto sino también la estrategia que lo acompaña y su nivel de adaptabilidad y evolución a lo largo del ciclo de vida (Santesmases, 1999).

Referirse específicamente al proceso de difusión y posicionamiento, remite al ejercicio que supone dar a conocer la existencia, características y ventajas del producto mediante una comunicación de tipo verbal y personal, a través de una red de vendedores, contando con los propios usuarios quienes transmiten conocimiento y experiencias a los no usuarios, o también mediante los medios de comunicación utilizando la publicidad y otros elementos derivados del uso de la información. En el proceso de difusión se destaca la posibilidad de adopción que 
consiste en la aceptación del nuevo producto por iniciativa propia -innovación- o como consecuencia de las experiencias obtenidas por otros consumidores -imitación-.

La comercialización hace referencia a la relación de intercambio que se establece entre la empresa y el mercado. Se considera como la última etapa del proceso no porque finalice la actividad, sino porque es allí precisamente, donde se pueden identificar necesidades del mercado que bien canalizadas permiten fortalecer el proceso productivo. Desde el punto de vista de la innovación es precisamentelacomercialización la que permite conectar efectivamente a la empresa con el mercado, para conocer sus necesidades y satisfacer sus demandas.

El seguimiento como parte del ejercicio de comercialización, permite monitorear el desempeño de la innovación y su progreso. Es una herramienta que sirve de punto de referencia para mejorar en el proceso de innovación y lograr niveles de efectividad cada vez mayores en el futuro. El monitoreo de la innovación como se le conoce técnicamente permite brindar un límite de tiempo para medir el progreso del esfuerzo innovador, ayuda a medir la distribución apropiada de los recursos orientados para tal fin, y se convierte en una herramienta de diagnóstico que puede emplearse para fijar áreas de problemas potenciales que pudieran necesitar ajuste y refuerzo (Kuczmarski, 1997).

Como se puede visualizar, en el proceso de innovación las ideas se generan a partir del reconocimiento de una necesidad y su posibilidad o viabilidad técnica pueden ser evaluadas antes de proceder. Solo se llega a la solución del problema luego de la búsqueda de información, la investigación y en general de un ejercicio constructivo altamente exigente. La innovación debe ser vista entonces, como un proceso necesario, útil y valioso, que permite sustentar la capacidad competitiva de cualquier idea de negocios. 